\title{
On the CVD Growth of C Nanotubes Over Fe-Loaded Montmorillonite Catalysts
}

\author{
Regular Paper
}

\author{
Saveria Santangelo ${ }^{1}$ Elpida Piperopoulos², Maurizio Lanza ${ }^{3, *}$, \\ Giuliana Faggio", Giacomo Messina ${ }^{1}$ and Candida Milone ${ }^{2}$ \\ 1 Department of Mechanics and Materials, University "Mediterranea", Italy \\ 2 Department of Industrial Chemistry and Materials Engineering, University of Messina, Italy \\ 3 CNR, Institute for Chemical Physical Processes, Messina Section, Italy \\ * Corresponding author E-mail: lanza@me.cnr.it
}

Received 18 July, 2011; Accepted 31 October, 2011

\begin{abstract}
The synthesis of carbon nanotubes (CNTs) by chemical vapor deposition (CVD) of isobutane $\left(i-\mathrm{C}_{4} \mathrm{H}_{10}\right)$ over sodium-exchanged K10-montmorillonite based ironcatalysts is investigated. By studying the influence of iron-addition (5-25 wt\%) on the catalyst performances, at $700^{\circ} \mathrm{C}$, an empirical relationship is derived relating the mass of CNTs synthesized with the exposed surface of loaded iron, as resulting from simultaneous change of number, size and dispersion of Fe-nanoparticles available for the growth.
\end{abstract}

Keywords Carbon nanotubes, Chemical vapor deposition, Isobutane, Montmorillonite-catalysts

\section{Introduction}

Laponite, clinoptilolite and montmorillonite are low-cost natural clay minerals. In the last years, they have been successfully utilized as catalyst support-materials for the production of CNTs, by CVD catalyzed by first-row transition metals [1-8], addressed to the development of binary hybrid nanocomposites to be used as polymeradditives for the improvement of the pristine polymer properties [2,9].
It has been recently demonstrated that iron loaded commercial montmorillonite (K10) behaves as a bifunctional catalyst in the CVD of $i-\mathrm{C}_{4} \mathrm{H}_{10}$ at $700{ }^{\circ} \mathrm{C}[7,8]$. Hydrocarbon decomposition occurring over added metal sites preferentially leads to the formation of multi-walled carbon nanotubes (MWCNTs), while Brönsted acid sites of the support are responsible for the formation of other carbon nanostructures, such as carbon nanofibers and disordered graphite flakes. The decomposition of hydrocarbon has been, hence, selectively driven towards MWCNTs by lowering the activity of support, via $\mathrm{Na}^{+}-$ exchange reaction or increase of catalyst reductiontemperature. Between the two different strategies the decrease of the support activity via $\mathrm{Na}^{+}$-exchange reaction allows obtaining, at given metal load, the highest amount of MWCNTs with improved crystalline quality $[7,8]$. Hybrid materials prepared by direct growth of MWCNTs over $\mathrm{Fe}-\mathrm{Na}^{+} / \mathrm{K} 10$ catalysts outstandingly enhance electrical conductivity of the insulating polylactide [9].

This work deals with the synthesis of MWCNTs by ironcatalyzed CVD over $\mathrm{Na}^{+}$-exchanged K10-montmorillonite based catalysts. It is a continuation and a completion of the previous study on this topic $[7,8]$. The influence of iron-addition (5-25 wt\%) on the catalyst performances is here investigated. Formerly studied un-exchanged K10- 
based catalysts are also considered and an empirical law is proposed to describe the growth of MWCNTs over added iron sites of both $\mathrm{Fe} / \mathrm{K} 10$ and $\mathrm{Fe} / \mathrm{Na}^{+}-\mathrm{K} 10$ catalysts.

\section{Experimental}

\subsection{Catalysts}

\subsubsection{Samples considered}

A set of five catalysts, based on sodium-exchanged K10montmorillonite, was prepared and characterized, as illustrated below. Besides, an additional set of three catalysts, based on un-exchanged commercial K10 clay, was considered. Their codes and specifics, as resulting from previous studies [7,8], are reported in Tab. 1 .

\subsubsection{Preparation}

Sodium-exchanged K10 montmorillonite was obtained by dispersing $10 \mathrm{~g}$ of as-purchased Aldrich K10 montmorillonite (size: $5-10 \mu \mathrm{m}$ ) in $300 \mathrm{ml}$ of water. Clay was allowed to swell by continuous stirring for about $6 \mathrm{~h}$. Then, water was replaced with $1 \mathrm{M}$ solution of $\mathrm{NaCl}$ to enable the ion-exchange reaction.

As-obtained $\mathrm{Na}^{+}-\mathrm{K} 10$ montmorillonite was used to prepare Fe-loaded catalysts. For this purpose, $5 \mathrm{~g}$ of unloaded $\mathrm{Na}^{+}-\mathrm{K} 10$ clay was impregnated with $8 \mathrm{ml}$ of an aqueous solution of $\mathrm{Fe}\left(\mathrm{NO}_{3}\right)_{3} \cdot 9 \mathrm{H}_{2} \mathrm{O}$ (Fluka, 99.9\%) having an amount of iron salt suitably calculated to obtain the desired extra Fe-load ( $\left.w_{\mathrm{L}}=5.0-25.0 \mathrm{wt} \%\right)$. Their codes, overall Fe-content $\left(w_{\mathrm{Fe}}\right)$ and specifics are reported in Tab. 1.

\subsubsection{Characterization}

NKw catalysts were slowly dried at $80^{\circ} \mathrm{C}$ and calcined at $450^{\circ} \mathrm{C}$ in air to get iron oxide from its precursor. Solids were well ground to powder and reduced for $2 \mathrm{~h}$ upon $120 \mathrm{cc} / \mathrm{min} 1: 1 \mathrm{H}_{2}+\mathrm{He}$ flow at $500^{\circ} \mathrm{C}\left(T_{\mathrm{R}}\right)$. The same route was previously followed to reduce $\mathrm{K} w \mathrm{~L}$ catalysts at $700^{\circ} \mathrm{C}$.
The specific surface area of reduced catalysts was determined by Brunauer-Emmett-Teller (BET) method using a Q-SURF Series Surface area analyzer. After outgassing the sample at $200^{\circ} \mathrm{C}$ for $2 \mathrm{~h}$ nitrogen was adsorbed at $-196^{\circ} \mathrm{C}$.

The phases present after reduction were investigated by performing x-ray diffraction (XRD) measurements. XRD data were collected with an APD 2000 Ital Structures diffractometer using $\mathrm{CuK} \alpha$ radiation source. The patterns were recorded in step scan mode (step: $0.02^{\circ}$ ) in the range $10-70^{\circ}$ of 2 -theta angles. Counting time was $1 \mathrm{sec} / \mathrm{step}$.

\subsection{Carbonaceous deposits}

\subsubsection{Synthesis and yield evaluation}

CVD growth reactions were carried out in $i-\mathrm{C}_{4} \mathrm{H}_{10}+\mathrm{H}_{2}$ atmosphere at $700^{\circ} \mathrm{C}$. The reduced catalyst $(0.5 \mathrm{~g})$ was placed in a quartz boat inside the quartz reactor, located in a horizontal electric furnace, and preliminarily heated upon $120 \mathrm{cc} / \mathrm{min}$ 1:1 $\mathrm{He}+\mathrm{H}_{2}$ flow up to synthesis temperature. Then helium was replaced with isobutane keeping constant flow ratio and total flow rate. After $2 \mathrm{~h}$ the reaction was stopped. The raw products were cooled down to room temperature (RT) in He atmosphere and weighed ( $0.1 \mathrm{mg}$ balance sensitivity) for yield evaluation.

The mass of deposited carbon $\left(m_{\mathrm{C}}=m_{\mathrm{T}}-m_{\mathrm{R}}\right)$ was estimated subtracting the mass $(m \mathrm{R})$ of reduced catalyst employed from the mass $(m \mathrm{~T})$ of materials (reaction products + catalyst) discharged from the reactor at the end of each synthesis. Two trials were carried for each parameter setting. Yield

$$
Y_{\mathrm{C}}=100 \cdot m \mathrm{C} / \mathrm{mR}_{\mathrm{R}}
$$

and specific yield

$$
S Y_{\mathrm{C}}=100 \cdot m \mathrm{C} / m_{\mathrm{Fe}}
$$

\begin{tabular}{|l|l|l|l|l|l|l|l|l|}
\hline Sample code & $\begin{array}{l}w_{\mathrm{Fe}} \\
(\mathrm{wt} \%)\end{array}$ & $\begin{array}{l}T_{\mathrm{R}} \\
\left({ }^{\circ} \mathrm{C}\right)\end{array}$ & $\begin{array}{l}A_{\mathrm{BET}} \\
\left(\mathrm{m}^{2} / \mathrm{g}\right)\end{array}$ & $\begin{array}{l}d_{\mathrm{Fe}} \\
(\mathrm{nm})\end{array}$ & $-\mathrm{F}$ & $\begin{array}{l}A_{\mathrm{Fe}} \\
\left(\mathrm{m}^{2} / \mathrm{g}\right)\end{array}$ & $\begin{array}{l}Y_{\mathrm{C}} \\
(\mathrm{wt} \%)\end{array}$ & $\begin{array}{l}S Y_{\mathrm{C}} \\
(\mathrm{wt} \%)\end{array}$ \\
\hline NK0 & 1.7 & 500 & 222 & $*$ & --- & --- & 3.0 & 176.5 \\
\hline NK5 & 6.7 & 500 & 164 & $*$ & --- & -- & 29.5 & 440.3 \\
\hline NK15 & 16.7 & 500 & 157 & 22.4 & $5.2 \times 10^{-2}$ & 5.1 & 179.9 & 1077.2 \\
\hline NK20 & 21.7 & 500 & 170 & 24.4 & $4.7 \times 10^{-2}$ & 6.3 & 217.8 & 1003.7 \\
\hline NK25 & 26.7 & 500 & 109 & 27.6 & $4.2 \times 10^{-2}$ & 6.9 & 207.0 & 775.3 \\
\hline K0 [7,8] & 2.0 & 700 & 201 & $*$ & --- & --- & 0.4 & 10.0 \\
\hline K5 $[7,8]$ & 7.0 & 700 & 115 & 32.0 & $3.6 \times 10^{-2}$ & 1.2 & 14.7 & 210.0 \\
\hline K15 $[7,8]$ & 17.0 & 700 & 69 & 45.1 & $2.6 \times 10^{-2}$ & 2.5 & 31.7 & 186.5 \\
\hline
\end{tabular}

Table 1. Codes, reduction temperature $\left(T_{\mathrm{R}}\right)$ and specifics of all the considered catalysts. Catalyst codes, NKwL and KwL, summarize information concerning the type of clay (NK and $\mathrm{K}$ stand for $\mathrm{Na}^{+}$-exchanged and un-exchanged $\mathrm{K} 10$ clay, respectively) and iron extra-load $(w \mathrm{~L}=5.0-25.0 \mathrm{wt} \%) . \mathrm{NK} 0$ and $\mathrm{K} 0$ denote unloaded catalysts. The overall metal content $\left(w_{\mathrm{Fe}}\right)$ is the sum of iron already contained in NK0 $(1.7 \mathrm{wt} \%)$ and $\mathrm{K} 0(2.0 \mathrm{wt} \%)$ and iron added by impregnation. $d_{\mathrm{Fe}}$ and $A$ вет denote mean size of Fe-particles and measured specific surface area of the overall catalysts (inclusive of metal and clay-support). A star marks cases in which $\mathrm{Fe}^{0}$ is not detected in XRD patterns. Mean catalytic yields $\left(Y_{\mathrm{C}}\right)$ and specific yields $\left(S_{\mathrm{C}}\right)$ are also reported, as well as calculated dispersion $(D)$ and specific surface area of iron $\left(A_{\mathrm{Fe}}\right)$. 
of the catalytic process were calculated by normalizing $m \mathrm{c}$ to the mass $\left(m_{\mathrm{R}}\right)$ of the whole catalyst (clay-support included) $[7,8,10,11]$ and to the mass $\left(m_{\mathrm{Fe}}\right)$ of its sole activecomponent. Mean values obtained are reported in Tab. 1.

\subsubsection{Purification and characterization}

Support and iron particles were subsequently removed by refluxing products obtained from reactions in a mixture of $12 \% \mathrm{HCl}$ and $12 \% \mathrm{HF}$ acids. After purification carbonaceous deposits (below coded as the catalyst used for their synthesis) were washed thoroughly with distilled water, dried at $110^{\circ} \mathrm{C}$ for $3 \mathrm{~h}$ and finally analyzed.

The process selectivity was evaluated by means of scanning electron microscopy (SEM) using JEOL JSM5600LV operating at $20 \mathrm{kV}$. Morphology, dimensions and crystalline structure of the MWCNTs synthesized were investigated by transmission electron microscopy (TEM) using JEOL JEM-2010, equipped with a Gatan 794 MultiScan CCD camera and operating at $200 \mathrm{kV}$. Several different SEM and TEM images with diverse magnification factors per specimen were recorded to have a reliable picture of the samples.

Their crystalline quality was evaluated by measuring Raman scattering excited by the $514.5 \mathrm{~nm}(2.41 \mathrm{eV})$ line of a Coherent Innova $70 \mathrm{Ar}^{+}$laser. The analysis was carried out in air at RT. Spectra were recorded in the 800$3350 \mathrm{~cm}^{-1}$ spectral range by using a Jobin Yvon Ramanor U-1000 double monochromator, equipped with an Olympus BX40 microscope (X50 objective) and a Hamamatsu R943-02 photomultiplier operating in photon-counting mode. The use of a low laser-power ( $3 \mathrm{~mW}$ at the sample surface) prevented annealing effects. In order to reliably describe the sample bulk, several different locations of each specimen were sampled on account of the possible structural non-homogeneity. A $30 \mathrm{~s}$ long acquisition time was used to improve the $\mathrm{S} / \mathrm{N}$ ratio. Spectra were normalized, averaged and finally reproduced by Lorentzian bands after constant background subtraction.

The Fe-impurity content and thermal stability of carbonaceous deposits were investigated by means of thermo-gravimetry (TG) using TA Instruments SDTQ 600 (0.1 $\mu \mathrm{g}$ balance sensitivity) and differential thermogravimetry (DTG). For this purpose, $2-3 \mathrm{mg}$ of each sample were analyzed. Each specimen, placed in a platinum pan, was firstly kept at $200^{\circ} \mathrm{C}$ in inert environment (argon) until balance stabilization and subsequently burned upon $100 \mathrm{cc} / \mathrm{min}$ pure-oxygen flow. The temperature range of $200-1000^{\circ} \mathrm{C}$ was explored at $1{ }^{\circ} \mathrm{C} / \mathrm{min}$ scan rate. A multipoint calibration was preliminarily carried out by means of metallic standards of very high purity.
Further technical details, concerning measurements performed and instrumentation utilized, can be found elsewhere [7,8,10-12].

\section{Results}

Figure 1 displays the XRD patterns of reduced catalysts with higher Fe-loads. The mean size of Fe-nanoparticles $\left(d_{\mathrm{Fe}}\right)$ is estimated via the Scherrer equation from the peak of metallic iron at 2 -theta $=44.7^{\circ}$. The results obtained are reported in Tab. 1 together with the overall specific surface area $($ Ввет) of the catalysts evaluated by BET method.

Figures $2-5$ show the results of the analyses performed on reaction products after purification. Figure 2 displays SEM micrographs of deposits obtained over Fe-loaded catalysts, while some TEM images are shown in Fig. 3. Results of TEM observations are summarized in Tab. 2 .

Figures4(a) and 4(b) display thermo-grams and differential mass-loss profiles. The relative amount of non-burnt matter at the end of each TG measurement [11] represents the content of Fe-impurities $\left(w_{\mathrm{I}}\right)$, while the maximum oxidation-rate temperature $\left(T_{\mathrm{P}}\right)$ in DTG profiles gives a measure of the thermal stability. The values of $w_{\text {I }}$ and $T_{\mathrm{P}}$ obtained are reported in Tab. 2 .

Figure 5 displays the shape evolution of the microRaman spectra. The main spectral features detected in all the samples investigated are the G-band at $1580 \mathrm{~cm}^{-1}$ (graphite-like in-plane optical mode associated to the stretching of all $C=C$ pairs), the $D$ band at $1350 \mathrm{~cm}^{-1}$ (forbidden in perfect graphite and rendered Raman-active by any kind of lattice defects

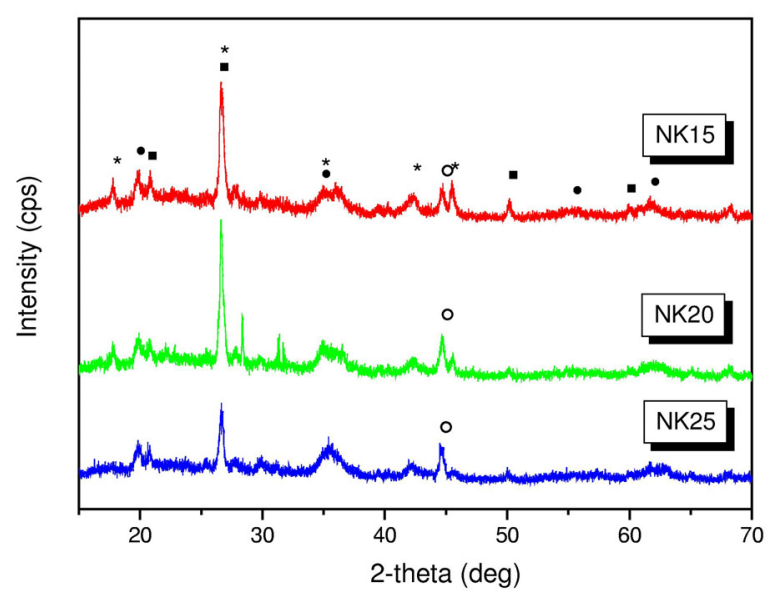

Figure 1. XRD patterns of Fe-loaded $\mathrm{Na}^{+}-\mathrm{K} 10$ catalysts with higher Fe-loads. Symbols: $(\bullet)$ montmorillonite, $\left(^{*}\right)$ muscovite, quartz and (o) metallic iron. 

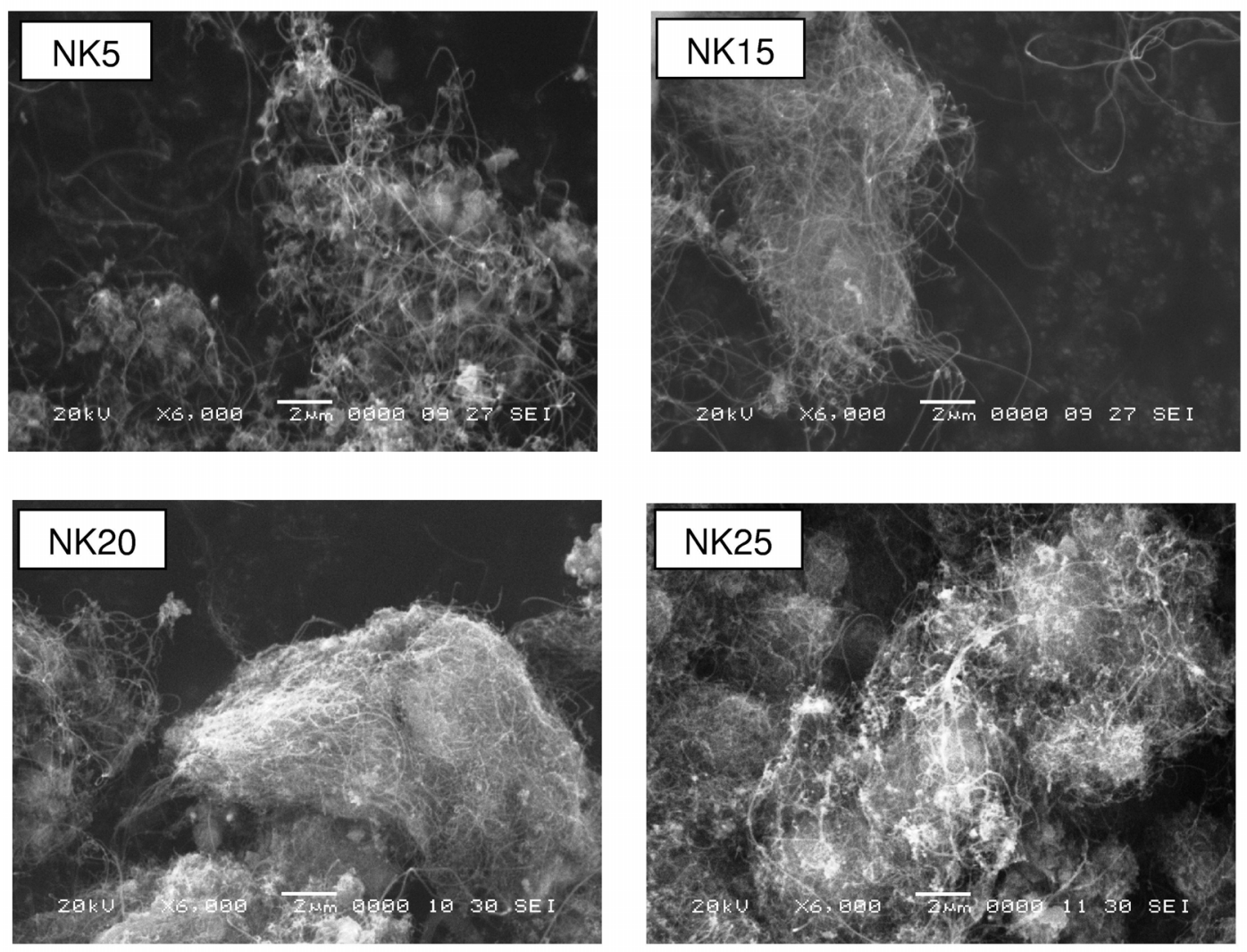

Figure 2. Selectivity towards nanotubes as monitored by SEM.
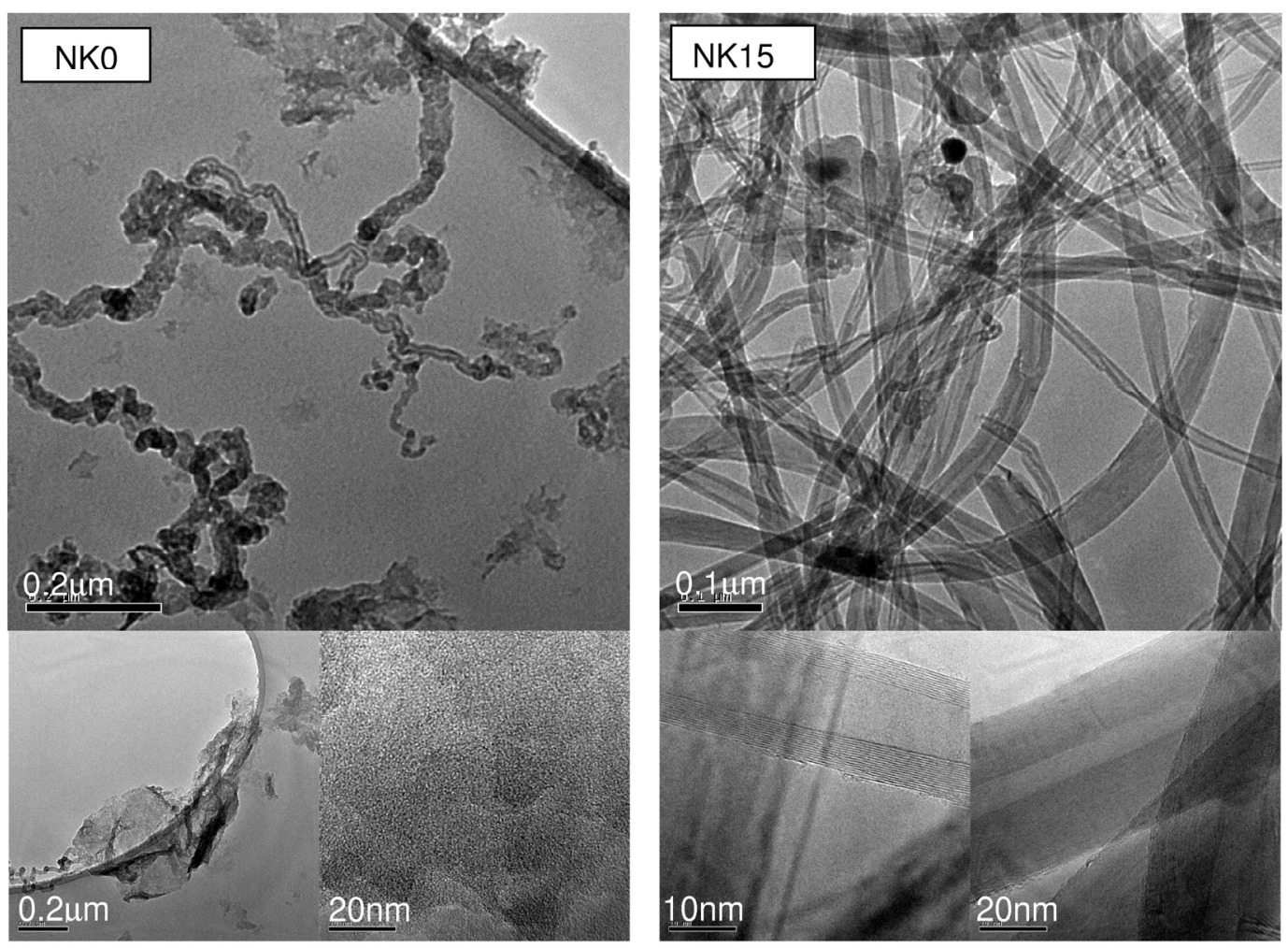

Figure 3. TEM micrographs of carbonaceous deposits synthesized over unloaded (NK0) and Fe-loaded $\mathrm{Na}^{+}-\mathrm{K} 10$ catalysts (the case of $15 \mathrm{wt} \% \mathrm{Fe}-\mathrm{load}$ is shown). Only nanofibers and disordered graphite flakes form on NK0. 

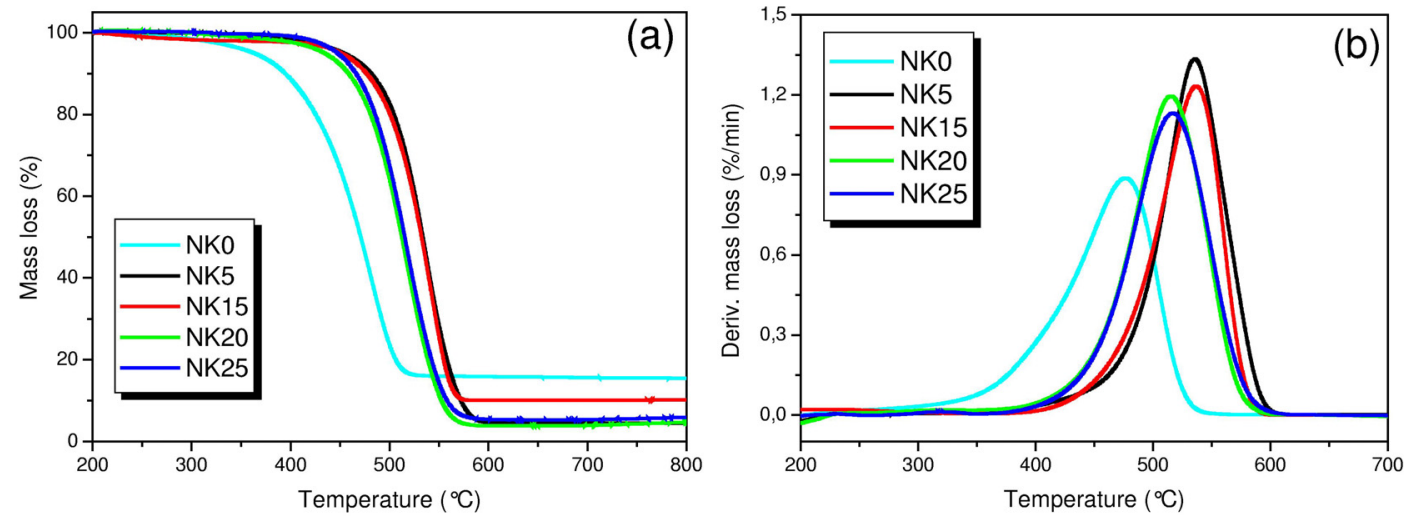

Figure 4. Results of (a) TG and (b) DTG analyses on purified C deposits.

relaxing the selection rules through the breaking of the basic symmetry of graphene-layers) and the $\mathrm{G}^{\prime}$-band at $2700 \mathrm{~cm}^{-1}$ (overtone of the D-band, Raman-allowed and defect-independent) [12-15].

The $G^{\prime} / D$ integrated intensity ratio $\left(I_{G^{\prime}} / I_{D}\right)$ gives a measure of the relative amount of the defect-free to ill-organized graphitic sheets and, hence, monitors the average crystalline quality (aromaticity) of the carbonaceous lattice $[8,10,12,15]$. The values obtained from the spectradecomposition are reported in Tab. 2 .

The G/D integrated intensity ratio $\left(I_{G} / I_{D}\right)$ is currently used to estimate the average size of crystallites $(L c)$ in all graphitic samples through empirical laws [16-19]. Recently, the relationship $L_{\mathrm{C}}=560 \cdot\left(I_{\mathrm{G}} / I_{\mathrm{D}}\right) \cdot E_{\mathrm{L}^{-4}}$, where $E_{\mathrm{L}}$ is the laser visible excitation energy $(2.41 \mathrm{eV}$, in present case) has been proposed for the estimation of $L_{c}$ in nanotubes and nanographites $[18,19]$. The values of $L c$ obtained by the use of this formula are reported in Tab. 2. Smaller $L_{c}$ values $(3.9-8.5 \mathrm{~nm})$ are attained by the use of the relationship $L_{\mathrm{C}}=4.4 \cdot\left(I_{\mathrm{G}} / I_{\mathrm{D}}\right)$, widely used in the past $[16,17]$.

\section{Discussion}

\subsection{Catalysts}

The diffraction peaks of $\mathrm{Na}^{+}-\mathrm{K} 10-$ montmorillonite clay [8] are detected in all the catalysts (Fig. 1). Structural analysis further reveals the presence of muscovite and quartz as clay's impurities. The peak of $\mathrm{Fe} 0$ is not detected in $\mathrm{NK} 0$ and NK5 catalysts [8], likely due to the insufficient amount of iron and/or to the smaller particle size. Instead, in the remaining catalysts (Fig. 1) its intensity progressively increases with the amount of metal loaded.

Also $d_{\mathrm{Fe}}$ slowly increases with Fe-load (Tab.1), causing also dispersion and exposed surface of the metal to vary.

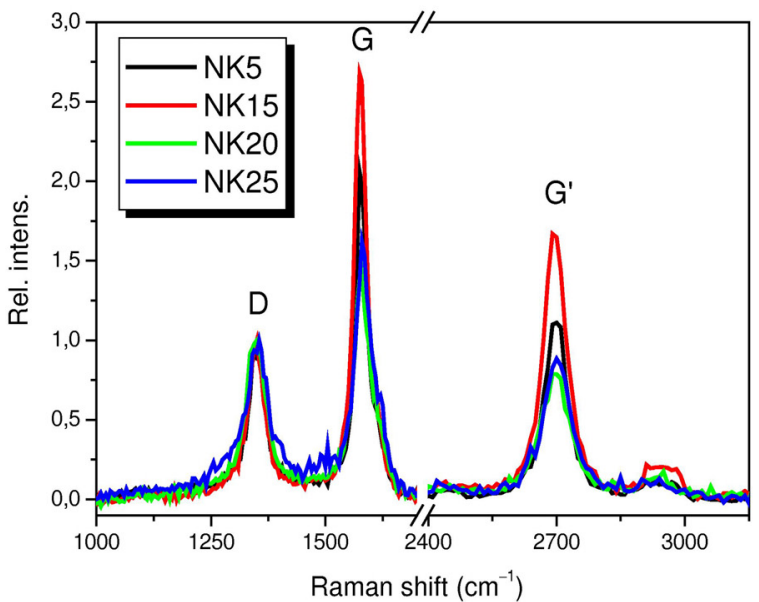

Figure 5. Results of MRS analysis on purified C deposits. Spectra are normalized to the D-band maximum intensity.

\begin{tabular}{|c|c|c|c|c|c|c|c|}
\hline \multirow[t]{2}{*}{ Sample code } & \multirow{2}{*}{$\begin{array}{l}\text { TEM } \\
\text { Morphology }\end{array}$} & \multirow{2}{*}{$\begin{array}{l}\text { TG } \\
w_{\mathrm{I}} \\
(\mathrm{wt} \%)\end{array}$} & \multirow{2}{*}{$\begin{array}{l}\text { DTG } \\
T_{\mathrm{P}} \\
\left({ }^{\circ} \mathrm{C}\right) \\
\end{array}$} & \multicolumn{4}{|l|}{ MRS } \\
\hline & & & & $I_{\mathrm{G}^{\prime}} / I_{\mathrm{D}}$ & $I_{\mathrm{G}} / I_{\mathrm{D}}$ & $\begin{array}{l}L c(a) \\
(\mathrm{nm})\end{array}$ & $\begin{array}{l}L c(b) \\
(\mathrm{nm})\end{array}$ \\
\hline NK0 & CNFs+DGFs & 16.6 & 475 & & & & \\
\hline NK5 & MWCNTs & 4.5 & 535 & 1.34 & 1.17 & 19.4 & 5.2 \\
\hline NK15 & MWCNTs & 10.0 & 536 & 2.43 & 1.93 & 32.0 & 8.5 \\
\hline NK20 & MWCNTs & 4.2 & 514 & 1.13 & 0.88 & 14.6 & 3.9 \\
\hline NK25 & MWCNTs & 5.5 & 515 & 0.99 & 0.88 & 14.6 & 3.9 \\
\hline
\end{tabular}

Table 2. Results of TEM, TG, DTG and MRS analyses carried out on purified reaction products. $w$ is the relative amount of Feimpurities present in C deposits. TP is peak temperature of DTG profiles giving a measure of thermal stability. The G'/D intensity ratio $\left(I_{G^{\prime}} / I_{D}\right)$ monitors crystalline quality. The G/D intensity ratio $\left(I_{\mathrm{G}} / I_{\mathrm{D}}\right)$ allows estimating the average size $(L c)$ of graphitic crystallites (the values calculated following both refs. [18,19] (a) and refs. [16,17] (b) are reported). 
Fe dispersion, namely the molar fraction of exposed iron, is calculated, assuming a spherical particle approximation, as [20]

$$
D=d \mathrm{VA} / d_{\mathrm{Fe}}=6 \tau \mathrm{Fe} /\left(a_{\mathrm{Fe}} \cdot d \mathrm{Fe}\right),
$$

where $d_{\mathrm{Fe}}$ is the mean metal particle size calculated via the Scherrer equation, $d_{V A}=6 \tau \mathrm{m} / a_{\mathrm{M}}$ is the volume-area mean diameter, $a_{\mathrm{M}}=6.13 \times 10^{-20} \mathrm{~m}^{2}$ is the effective average area occupied by a metal atom in the surface [20] and $\tau_{\mathrm{M}}=1.18 \times 10^{-29} \mathrm{~m}^{3}$ is the volume per metal atom in the bulk, calculated as $w /\left(N_{\mathrm{A}} \cdot \rho\right)$, with $N_{\mathrm{A}}=6.023 \times 10^{23} / \mathrm{mol}$ standing for the Avogadro's number, $w=55.845$ a.m.u. denoting the atomic weight of iron and $\rho=7.86 \times 10^{6} \mathrm{~g} / \mathrm{m}^{3}$ being the mass density of bulk iron.

By this procedure, $D$ is found to decrease from $5.2 \times 10^{-2}$ to $4.2 \times 10^{-2}$ going from sample NK15 to NK25 (Tab.1). The same calculation is applied to the formerly studied unexchanged $\mathrm{K} 10$-catalysts reduced at $700^{\circ} \mathrm{C}$. Lower $\mathrm{D}$ values (Tab. 1) are obtained for catalysts $\mathrm{K} 5$ and $\mathrm{K} 15$, based on $d_{\mathrm{Fe}}$ data inferred from previous XRD measurements [8]. Specific surface area

$$
A_{\mathrm{Fe}}=D \cdot a_{\mathrm{M}} \cdot N_{\mathrm{A}} \cdot n_{\mathrm{Fe}}
$$

(where $n_{\mathrm{Fe}}$ is the overall number of moles of added iron per gram of catalyst) and surface area

$$
S_{\mathrm{Fe}}=A_{\mathrm{Fe}} \cdot m_{\mathrm{R}}
$$

of the exposed iron are found to slightly increase, respectively from $5.1 \mathrm{~m}^{2} / \mathrm{g}$ to $6.9 \mathrm{~m}^{2} / \mathrm{g}$ (Tab.1) and from $2.6 \mathrm{~m}^{2} / \mathrm{g}$ to $3.5 \mathrm{~m}^{2} / \mathrm{g}$, going from sample NK15 to NK25 (Tab. 1). Smaller $A_{\mathrm{Fe}}$ (Tab.1) and $S_{\mathrm{Fe}}$ values are obtained for catalysts $\mathrm{K} 5$ and $\mathrm{K} 15$.

It is worthwhile noting that in all the cases the calculated specific surface area of the metal is always much smaller than the overall specific surface area, Aвет, of the catalysts (Tab.1), demonstrating that iron does not completely cover the clay surface, either at higher loads.

\subsection{Carbon deposits}

TEM analysis had previously evidenced $[7,8]$ that only carbon nanofibers (CNFs) and disordered graphite flakes (DGFs) form on the unloaded clay. Present study (Fig. 3) reveals that MWCNTs are, instead, obtained over all the Fe-loaded catalysts, regardless their load. SEM analysis (Fig. 2) evidences no substantial change in their morphology, but increased entanglement of the tubes and less uniform and larger diameters, in accord with the increase of $d_{\mathrm{Fe}}[21,22]$.

Disordered carbon nanostructures formed over NK0 are here found to contain the highest amount of Fe-impurities $\left(w_{\mathrm{I}}=17 \mathrm{wt} \%\right)$. In addition, as lattice defects act as initial oxidation sites [23], they also possess the lowest thermal stability $\left(T_{\mathrm{P}}=475^{\circ} \mathrm{C}\right)$.

Among MWCNTs, the highest oxidative resistance $\left(T_{\mathrm{P}}=536^{\circ} \mathrm{C}\right)$ pertains to sample NK15, where TEM analysis reveals the presence of straight and smooth tubes consisting of regular sequence of perfectly ordered graphitic layers (Fig. 3). Very intense G- and G'-bands dominate the Raman spectrum of this sample (Fig. 5), whose crystalline quality $\left(I_{\mathrm{G}^{\prime}} / I_{\mathrm{D}}=2.43\right)$ largely exceeds that of remaining samples (Tab.2), as well as that of MWCNTs reported as the best crystallized ever obtained over clay-based catalysts [5,7]. The high crystalline perfection of MWCNTs NK15 is proved also by the size of crystallites $(32 \mathrm{~nm})$, larger by a factor of 1.5-2.0 than in remaining NKwL samples (Tab. 2).

Recent studies have then demonstrated that, thanks to the high graphization-degree of these MWCNTs, the incorporation of small amounts $(1-5 \mathrm{wt} \%)$ of hybrid NK15 (MWCNTs + clay) within an insulating polymeric matrix outstandingly enhances its electrical conductivity (up to 9 orders of magnitude) with respect to the pristine polymer [9].

\subsection{Growth process over clay-based catalysts}

As shown in Tab.1, at lower loads, $Y_{C}$ rapidly increases with $\mathrm{Fe}$ addition. The maximum value $(\sim 2.2$ grams of deposited carbon per gram of catalyst) is reached for $w_{\mathrm{L}}=20 \mathrm{wt} \%$. Above this load $Y_{\mathrm{C}}$ decreases, in spite the larger amount of iron. This is because $S Y_{C}$ progressively drops above $w_{\mathrm{L}}=15 \mathrm{wt} \%$, load for which the largest amount ( 12 grams) of carbon per gram of iron is produced.

As initially mentioned, previous studies demonstrated that lowering the acidity of the clay support, via $\mathrm{Na}^{+}$ exchange reaction or reduction-temperature enhancement, inhibits the formation of highly disordered carbonaceous nanostructures, responsible for metal deactivation at small iron loads $\left(w_{\mathrm{L}}=5 \mathrm{wt} \%\right)$, producing beneficial effects on yield [7].

For $w_{\mathrm{L}} \leq 15 \mathrm{wt} \%, Y_{\mathrm{C}}$ was shown to effectively increase exponentially with the product $w_{\mathrm{Fe}} \cdot A$ вет in un-exchanged $\mathrm{K} 10$ catalysts reduced at $700^{\circ} \mathrm{C}$ [7], as well as in $\mathrm{Na}^{+}$exchanged $\mathrm{K} 10$ catalysts reduced at lower temperature $\left(500^{\circ} \mathrm{C}\right)$ as in present study. A saturating trend is here observed at higher loads [Fig. 6(a)].

The same trend is observed when $m c$ is plotted against the exposed surface of loaded iron SFe [Fig.6(a)]. Note that this regards exclusively samples (NK15-NK25 and $\mathrm{K} 5-\mathrm{K} 15)$ for which $d_{\mathrm{Fe}}$ data are available. As a fixed amount of reduced catalyst is here used, based eq. (1.a), 

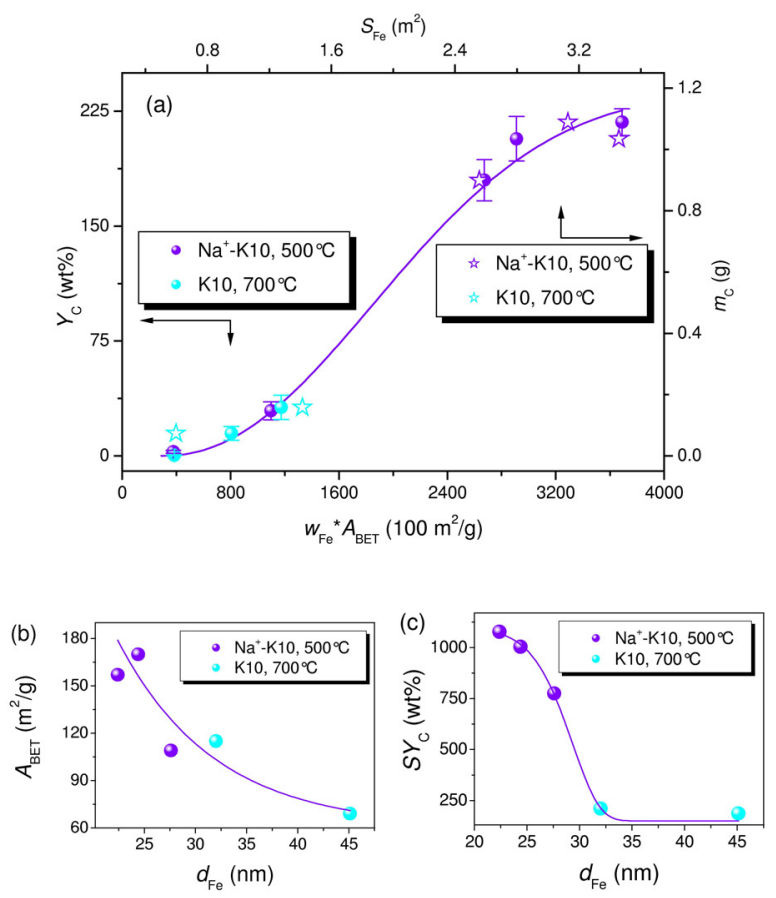

Figure 6. (a) Comparison between plots of $Y_{C} v s$. the product $w_{\text {Fe }} \cdot A$ BET and of $m$ C $v s$. SFe for $\mathrm{Na}^{+}-\mathrm{K} 10$ catalysts (present study) and K10 catalysts [7] reduced at different temperature (arrows indicate which axes the data refer to; length of error bars equals the difference between $Y_{C}$ values obtained in the two trials). The dependence of (b) $A_{\text {вет }}$ and (c) $S Y_{\mathrm{C}}$ on $d_{\mathrm{Fe}}$ is also shown.

$m_{\mathrm{c}}$ is proportional to $Y_{\mathrm{C}}$. Therefore, the similarity between $Y_{\mathrm{C}} v s$ wFe-ABET and $m \mathrm{C}$ vs $S_{\mathrm{Fe}}$ trends hints at the existence of a linear relationship between $S_{\mathrm{Fe}}$ and $w_{\mathrm{Fe}} \cdot A_{\mathrm{BET}}$, as actually found. In fact, $A_{\mathrm{BET}}$ is here found to coarsely scale as $1 / d_{\mathrm{Fe}}$ [Fig. 6(b)]. For fixed $m_{\mathrm{R}}=0.5 \mathrm{~g}, S_{\mathrm{Fe}}$ is proportional to $A_{\mathrm{Fe}}$ [eq. (3.b)]. In turn, according to eq. (3.a), $A_{\mathrm{Fe}} \propto D \cdot n_{\mathrm{Fe}}$, where of course $n_{\mathrm{Fe}} \propto w_{\mathrm{Fe}}$ and, based on eq. (2), $D \propto 1 / d_{\mathrm{Fe}}$ just alike $A$ вет.

Both these findings deserve a detailed and attentive discussion in order to avoid drawing erroneous conclusions.

An important consequence of the existence of the effective dependence of $Y_{\mathrm{C}}$ on $w_{\mathrm{Fe}} \cdot A_{\mathrm{BET}}$ is that, in $\mathrm{K} w \mathrm{~L}$ catalysts reduced at $700{ }^{\circ} \mathrm{C}$, a higher $w_{\mathrm{Fe}}$ may compensate for a lower $A$ вет to finally give the same (or comparable) $w_{\mathrm{Fe}} \cdot A \mathrm{BET}$ product as in $\mathrm{NK} w_{\mathrm{L}}$ catalysts reduced at $500^{\circ} \mathrm{C}$, hence resulting in the same (or comparable) $Y_{\mathrm{C}}$. This is immediately evident by considering the values of $w \mathrm{Fe}$, $A_{\text {вEт }}$ and $Y_{\mathrm{C}}$ relative to samples NK5 and K15 reported in Tab. 1 and/or by comparing the corresponding data in Fig.6(a). As can be seen, as strictly concerns catalytic yield, in spite of different $w_{\mathrm{Fe}}$ and $T_{\mathrm{R}}$ (and resulting $n_{\mathrm{Fe}}$ and $d_{\mathrm{Fe}}$ ) the two catalysts behave similarly.

As for the found dependence of $m \mathrm{c}$ on $S_{\mathrm{Fe}}$, it can be argued that, for catalysts NK15-NK25 and K5-K15, the amount of produced carbon is ultimately determined exclusively by the exposed surface of added iron, as evaluated [through eqs. (3.a) and (3.b)] on the basis of merely geometric factors ( $d_{\mathrm{Fe}}$ and resulting $D$ ) besides to, of course, the iron content $(n \mathrm{Fe})$ and the catalyst amount $(m \mathrm{R})$.

This is not a surprising result because all measured $d_{\mathrm{Fe}}$ values largely exceed $10 \mathrm{~nm}$ (Tab.1), value above which the relative amount of the diverse types of iron atoms (saturated ones, present in the crystal face, and unsaturated ones, present at edges, corners and terraces), endowed with different catalytic activity, hardly changes with size [20].

Based on the similar trends of the curves $Y_{\mathrm{C}}\left(w_{\mathrm{Fe}} \cdot A_{\mathrm{BET}}\right)$ and $m_{\mathrm{C}}\left(\mathrm{S}_{\mathrm{Fe}}\right)$ [Fig.6(a)], one might be brought to extend considerations made for NK15-NK25 and K5-K15 to the remaining samples. Indeed, the lack of information about $d_{\mathrm{Fe}}$ impedes any straight widening of the above discussion. In fact, for $d_{\mathrm{Fe}}<10 \mathrm{~nm}$, also the different catalytic activity and relative amount of the diverse types of iron atoms should be taken into account.

By fitting 10 available experimental data, it is found that the mass $m c$ of nanotubes, synthesized at $700{ }^{\circ} \mathrm{C}$ upon $(120 \mathrm{cc} / \mathrm{min})$ 1:1 $i-\mathrm{C}_{4} \mathrm{H}_{10}+\mathrm{H}_{2}$ flow over $(0.5 \mathrm{~g}) \mathrm{Na}^{+}-\mathrm{K} 10$ and K10 iron catalysts, increases with the exposed surface of loaded iron $S_{\mathrm{Fe}}$ according to the relationship

$$
m_{\mathrm{C}}=1.09-1.07 \cdot \exp \left(-\left(\left(S_{\mathrm{Fe}}+5.50\right) / 7.74\right)^{12.9}\right) .
$$

Although the choice of the function to be utilized to reproduce $m c$ data of Fig. 6(a) is to some extent arbitrary, that here used is theoretically inferred from the simple physical model below.

To this purpose, it is worthwhile noting that, far from establishing a general model of the growth process, the discussion below aims exclusively at accounting for the changes of $m c$ with $S_{\mathrm{Fe}}$ observed in the samples under study.

Therefore, a stringent simplifying assumption is introduced, consisting in attributing the same active surface to both the kinds of unloaded catalysts $\left(\mathrm{Na}^{+}-\right.$ exchanged $\mathrm{K} 10$ reduced at $500^{\circ} \mathrm{C}$ and un-exchanged $\mathrm{K} 10$ reduced at $\left.700^{\circ} \mathrm{C}\right)$.

The main reason for this choice is that very large errors affect lower $Y_{\mathrm{C}}$ and $m \mathrm{c}$ values (relative error is well above $75 \%$ in case of $\mathrm{K} 0$ catalyst), which renders them hardly distinguishable, at least as concerns the present aim of reproducing the changes of $m \mathrm{c}$ with $S_{\mathrm{Fe}}$.

Actually, unloaded catalysts exhibit comparable iron contents (Tab.1), but the diverse $T_{\mathrm{R}}$ might introduce a 
relevant size-difference. However, the very small reduction of the pristine iron content $\left(\Delta w_{\mathrm{Fe}}=-0.3 \mathrm{wt} \%\right.$, Tab.1), produced by the ion exchange reaction with a $\mathrm{NaCl}$ solution, suggests that Fe cation is not in an exchangeable position, but it is mainly present as a structural ion and/or as iron oxide formed from the dissolution of octahedral iron sites upon acid treatment [8]. This hints at a strong iron/support interaction, which likely hampers, or at least severely limits, possible sintering effects and size-variations at higher $T_{\mathrm{R}}$. Indeed, Aвет, which, as shown [Fig.6(b)], is sensitive to the changes of $d_{\mathrm{Fe}}$, exhibits quite similar values in the two unloaded catalysts (Tab. 1).

Thus, indicated with $S_{0}$ the active surface in unloaded catalysts and with $S_{\mathrm{Fe}}$ the surface increase brought about by iron addition, let $S=S_{0}+S_{\mathrm{Fe}}$ be the overall iron exposedsurface and $m c$ the mass of carbon correspondingly synthesized.

Because of the low fraction of supplied carbon that is transformed to carbon deposits (maximum: $6.8 \mathrm{wt} \%$ after $2 \mathrm{~h}$ of reaction for $w_{\mathrm{L}}=20 \mathrm{wt} \%$ ), the incident $\mathrm{C}$ flux does not suffer significant impoverishment owing to the formation of carbonaceous products. Thus, in principle, it should be expected that the increase of $S$ with $w$ L would produce a linear increase of $m c$. Nonetheless, it has to be borne in mind that the increase of $S$ with $w$ L is the result of the simultaneous variation of manifold influential factors (amount, size and related dispersion of Fenanoparticles, possibly involving also textural effects) and that an optimal size-range relative to C supply [24,25] does exist, as demonstrated by the dependence of $S Y_{C}$ on $d_{\text {Fe }}$ shown in Fig. 6(c).

Based on all these factors, a non-linear dependence of $m \mathrm{c}$ on $S$ can be reasonably hypothesized.

Therefore, if the variation, $\mathrm{d} m \mathrm{c}$, undergone by $m \mathrm{c}$ for an increment of the effective-variable $\mathrm{d}\left(S^{\alpha}\right)$, is taken as proportional, according to the simplest possibility, besides to $\mathrm{d}\left(S^{\alpha}\right)$, to the residual "distance" $\left(m \mathrm{c}^{\text {max }}-m \mathrm{c}\right)$ from the largest mass amount, $m c^{\max }=1.09 \pm 0.04 \mathrm{~g}$ (where the error is evaluated considering the different $Y_{C}$ values obtained in the two trials carried out upon fixed conditions), which is produced under the considered growth conditions, namely

$$
\mathrm{d} m \mathrm{c} \propto\left(m \mathrm{c}^{\max }-m \mathrm{c}\left(S^{\alpha}\right)\right) \cdot \mathrm{d}\left(S^{\alpha}\right),
$$

by setting $X=m \mathrm{c}^{\max }-m c$, the differential equation $\mathrm{d} X=-a \cdot X \cdot \mathrm{d}\left(S^{\alpha}\right)$ is obtained that can be solved in terms of $X$ to give

$$
X=A \cdot \exp \left(-\left(S / S^{*}\right)^{\alpha}\right)
$$

This finally becomes

$$
m \mathrm{c}=m \mathrm{c}^{\mathrm{max}}-A \cdot \exp \left(-\left(\left(S_{0}+S_{\mathrm{Fe}}\right) / S^{*}\right)^{\alpha}\right),
$$

where $S^{*}$ is a critical $S$ value. This expression, besides accounting for the positive curvature of the $m c\left(S_{\mathrm{Fe}}\right)$ curve at small $S_{F e}$ (Fig. 3), is also able to reproduce the negative curvature and saturating trend at larger $S_{\mathrm{Fe}}$. As the experimental data are fitted to this function, $A=1.07 \pm 0.02 \mathrm{~g}$, $S_{0}=5.50 \pm 0.04 \mathrm{~m}^{2}, S^{*}=7.74 \pm 0.04 \mathrm{~m}^{2}$ and $\alpha=12.9 \pm 0.6$ are found, leading to eq. (4) and validating the quite simple assumptions made. Empirical law (4) describes how the amount of grown MWCNTs increases with the overall exposed surface of iron, and includes the effect of the simultaneous variation of number, size and dispersion of Fe-nanoparticles available for their growth, as well as of the existence of an optimal size-range relative to $C$ supply $[24,25]$.

In case of mono-dispersed iron (namely, $D=1$ ), eq. (2) gives $d_{\mathrm{Fe}}=d_{\mathrm{VA}}=1.2 \mathrm{~nm}$ that is the diameter of "onlysurface-atom cluster" containing ca 100 atoms [20]. Interestingly, assuming that in the unloaded catalyst iron is nearly mono-dispersed $(D \leq 1)$ and accordingly using $d_{\mathrm{Fe}} \geq d_{\mathrm{VA}}$ as mean Fe-nanoparticle size value to calculate specific surface area of the exposed iron, from eq. (3.a) $A_{\mathrm{Fe}} \leq 11.24 \mathrm{~m}^{2} / \mathrm{g}$ is obtained. According to eq. (3.b), being $m_{\mathrm{R}}=0.5 \mathrm{~g}$, this corresponds to $S_{0} \leq 5.62 \mathrm{~m}^{2}$, value which is fairly compatible with the one $\left(5.50 \mathrm{~m}^{2}\right)$ derived from the fitting procedure.

Also the large value of the exponent $\alpha$ (12.9) needs a brief comment. Combining eqs. (1.a) and (1.b) catalytic yield can be written as $Y_{\mathrm{C}}=w_{\mathrm{Fe}} \cdot S Y_{\mathrm{C}} / 100$, so as $m_{\mathrm{C}}=w_{\mathrm{Fe}} \cdot S Y_{\mathrm{C}} \cdot m_{\mathrm{r}}$. Thus, the observed (non linear) dependence of $m \mathrm{c}$ on $S_{\mathrm{Fe}}$ [i.e. eq. (4)] is through $w_{\mathrm{Fe}}$ and $S Y_{\mathrm{C}}$. Different from $w_{\mathrm{Fe}}$, whose changes cause a smooth variation of $d_{\mathrm{Fe}}$ $\left(22.4-27.6 \mathrm{~nm}\right.$ at $500{ }^{\circ} \mathrm{C}$ and $32.0-45 \mathrm{~nm}$ at $700^{\circ} \mathrm{C}$ ) and, hence, of $S_{\mathrm{Fe}} S Y_{\mathrm{C}}$ is strongly sensitive to the changes of $d_{\mathrm{Fe}}$ and drastically declines at larger $d_{\mathrm{Fe}}$ [Fig. 6(c)].

\section{Conclusion}

The growth of MWCNTs by isobutane decomposition at $700^{\circ} \mathrm{C}$ is considered. The influence of iron-addition $(0$ $25 \mathrm{wt} \%$ ) on the performances of $\mathrm{Na}^{+}-\mathrm{K} 10$ based catalysts is investigated. For this purpose, XRD and BET measurements on reduced catalysts and SEM, TEM, TG and MRS analyses on purified carbon deposits are carried out.

The comparison of present results with those formerly obtained by the use of $\mathrm{Fe} / \mathrm{K} 10$ catalysts allows clarifying the physical meaning of the previously found effective dependence of yield $Y_{\mathrm{C}}$ on the product, $w_{\mathrm{Fe}} \cdot A_{\mathrm{BET}}$, of overall iron-content $w_{\mathrm{Fe}}$ times overall specific surface area $A_{\text {вет }}$ of the catalysts. The empirical law reflects the increase in the amount of grown MWCNTs produced by that of the exposed surface of iron, as a purely geometric effect, and includes the effect of the simultaneous 
variation of number, size and dispersion of Fenanoparticles available for their growth, as well as of the existence of an optimal size-range relative to the fixed $\mathrm{C}$ supply.

\section{References}

[1] K. Hernadi, "Catalytic Synthesis of Multiwall Carbon Nanotubes from Methylacetylene", Chem. Phys. Lett., vol. 363, pp. 169-174, 2002.

[2] D. Gournis, M. A. Karakassides, T. Bakas, N. Boukos, and D. Petridis, "Catalytic Synthesis of Carbon Nanotubes on Clay Minerals", Carbon vol. 40, pp. 2641-2646, 2003.

[3] A. Bakandritsos, A. Simopoulos, and D. Petridis, "Growth of Carbon Nanotubes on a Swellable Clay matrix", Chem. Mater., vol. 17, pp. 3468-3474, 2005.

[4] A. Bakandritsos, A. Simopoulos, and D. Petridis, "Iron Changes in Natural and Fe(III) Loaded Montmorillonite during Carbon Nanotube Growth", Nanotechnology, vol. 17, pp. 1112-1117, 2006.

[5] T. Tsoufis, L. Jankovic, D. Gournis, P. N. Trikalitis, and T. Bakas, "Evaluation of First-Row Transition Metal Oxides Supported on Clay Minerals for Catalytic Growth of Carbon Nanostructures", Mater. Sci. Eng., B, vol. 152, pp. 44-49, 2008.

[6] M. Kadlečíková, J. Breza, K. Jesenák, K. Pastorková, V. Luptáková, M. Kolmačka, A. Vojačková, M. Michalka, I. Vávra, and Z. Križanová, "The Growth of Carbon Nanotubes on Montmorillonite and Zeolite (Clinoptilolite)", Appl. Surf. Sci., vol. 254, pp. 5073-5079, 2008.

[7] S. Santangelo, M. Dhanagopal, G. Faggio, G. Messina, A. Pistone, M. Lanza, and C. Milone, "Preparation of Nanotubes-Clay Hybrid Systems by Iron-Catalyzed Isobutane Decomposition”, Diamond Relat. Mater., vol. 19, pp. 599-604, 2010.

[8] C. Milone, M. Dhanagopal, S. Santangelo, M. Lanza, S. Galvagno, and G. Messina, "Growth of Multi Walled Carbon Nanotubes through CCVD over FeLoaded K10 Montmorillonite Catalysts", Ind. Eng. Chem. Res., vol. 49, pp. 3242-3249, 2010.

[9] S. Santangelo, G. Gorrasi, R. Di Lieto, S. DePasquale, G. Patimo, E. Piperopoulos, M. Lanza, G. Faggio, F. Mauriello, G. Messina, and C. Milone, "Polylactide and Carbon Nanotubes/Smectite-Clay Nanocomposites: Preparation, Characterization, Sorptive and Electrical Properties", Appl. Clay Sci., Thematic Issue "Clay-Based Catalysts and Catalysis", DOI: 10.1016/j.clay.2010.12.013

[10] G. Messina, V. Modafferi, S. Santangelo, P. Tripodi, M.G. Donato, M. Lanza, S.Galvagno, C. Milone, E. Piperopoulos, and A. Pistone, "Large-Scale Production of High-Quality Multi-Walled Carbon Nanotubes: Role of Precursor Gas and of Fe-Catalyst Support", Diamond Relat. Mater., vol. 17, pp. 14821488, 2008.
[11] S. Santangelo, G. Messina, M. G. Donato, M. Lanza, C. Milone, and A. Pistone, "Low-Frequency Raman Study of Hollow Multi-Walled Nanotubes Grown by Fe-Catalyzed Chemical Vapor Deposition", J. Appl. Phys., vol. 100, pp. 104311-104315, 2006.

[12] S. Santangelo, G. Messina, G. Faggio, M. Lanza, and C. Milone, "Evaluation of Crystalline Perfection Degree of Multi-Walled Carbon Nanotubes: Correlations between Thermal Kinetic Analysis and Micro-Raman Spectroscopy", J. Raman Spectrosc., vol. 42, pp. 593-602, 2011.

[13] H. Kuzmany, B. Burger, A. Thess, and R. Smalley, "Vibrational Spectra of Single Wall Carbon Nanotubes", Carbon, vol. 36, pp. 709-712, 1998.

[14] M. S. Dresselhaus, G. Dresselhaus, R. Saito, and A. Jorio, "Raman Spectroscopy of Carbon Nanotubes", Phys. Rep., vol. 409, pp. 47-99, 2005.

[15] R. A. DiLeo, B. J. Landi, and R. P. Raffaelle, “Purity Assessment of Multi Walled Carbon Nanotubesby Raman Spectroscopy", J. Appl. Phys., vol.101, pp. 64307-64311, 2007.

[16] F. Tuinstra, and J. L. Koening, "Raman Spectrum of Graphite", J. Chem. Phys., vol. 53, pp. 1126-1130, 1970.

[17] D. S. Knight, and W. White, "Characterization of Diamond films by Raman Spectroscopy", J. Mater. Res., vol. 4, pp. 385-393, 1989.

[18] L. G. Cançado, K. Takai, T. Enoki, M. Endo, Y. A. Kim, H. Mizusaki, A. Jorio, L. N. Coelho, R. Magalhães-Paniago, and M. A. Pimenta, "General Equation for the Determination of the Crystallite Size LA of Nanographite by Raman Spectroscopy", Appl. Phys. Lett., vol. 88, pp. 163106-3, 2006.

[19] M. A. Pimenta, A. P. Gomes, C. Fantini, L. G. Cançado, P. T. Araujo, I. O. Maciel, A. P. Santos, C. A. Furtado, V. S. T. Peressinotto, F. Plentz, and A. Jorio, “Optical Studies of Carbon Nanotubes and Nanographites", Physica E, vol. 37 pp. 88-92, 2007.

[20] J. R. Anderson, “Measurement Techniques: Surface Area, Particle Size and Pore Structure" in Structure of metal catalysts, Academic Press Inc, London, UK, 1975, Chpt. 6, pp. 296 and 360.

[21] C. Ducati, I. Alexandou, M. Chhowalla, J. Robertson, and G. A. Amaratunga, "The Role of the Catalytic Particle in the Growth of Carbon Nanotubes by Plasma Enhanced Chemical Vapor Deposition", J. Appl. Phys., vol. 95, pp. 6387-6391, 2004.

[22] C. N. R. Rao, B. C. Satishkumar, A. Govindaraj, M. Nath, "Nanotubes". ChemPhysChem, vol. 2, pp. 78105, 2001.

[23] P. M. Ajayan, T. W. Ebbesen, T. Ichihashi, S. Iijima, K. Tanigaki, and H. Hiura, "Opening Carbon Nanotubes with Oxygen and Implications for Filling", Nature, vol. 362, pp. 522-525, 1993. 
[24] C. Ducati, I. Alexandou, M. Chhowalla, G. A. Amaratunga, and J. Robertson, "Temperature Selective Growth of Carbon Nanotubes by Chemical Vapor Deposition", J. Appl. Phys., vol. 92, pp. 3299-3303, 2002.

[25] S. Santangelo, G. Messina,G. Faggio, M. Lanza, A. Pistone, and C. Milone, "Calibration of Reaction Parameters for the Improvement of Thermal Stability and Crystalline Quality of Multi-Walled Carbon Nanotubes", J. Mater. Sci., vol.45, pp. 783-792, 2010. 